\title{
Vitamin D, Oxidative Stress and Cognition in Diabetes Mellitus
}

\author{
Kanchana R. ${ }^{1}$, Pushpa K. ${ }^{2}$ \\ ${ }^{1}$ Assistant Professor in Physiology, Dr. Chandramma Dayananda Sagar Institute of Medical Education and \\ Research, Harohalli, ${ }^{2}$ Associate Professor in Physiology, Sridevi Institute of Medical Sciences and Research \\ Hospital, Tumkur
}

\begin{abstract}
Introduction: Diabetes is a very common disease that affects almost all body systems. Of late recent studies have determined that vitamin D deficiency can cause several diseases such as diabetes, cardiovascular complications etc. Oxidative stress is an imbalance between free radicals and antioxidants in the body. Vitamin D is an antioxidant and its deficiency can cause oxidative stress . Thus vitamin D deficiency by itself and by causing oxidative stress can increase risk of developing diabetes
\end{abstract}

Hyperglycemia can affect cognition. Oxidative stress too can affect cognition. Studies done studying the role of vitamin D affecting cognition especially in diabetics are very few.

Aim: To determine the role of vitamin D in affecting cognition in Diabetic patients. To determine the correlation between vitamin $\mathrm{D}$, oxidative stress and cognition in diabetes .

Materials and Method:

Comparative cross sectional study

100 Diabetes patients were studied.

Exclusion Criteria: Hypertensives, alzheimers disease, dementia, bone diseases, epileptics, taking calcium or vitamin d supplements, hypo or hyperparathyroidism, thyroid disorders.

After obtaining ethical approval from the institution a structured questionnaire was given to all.

Fasting blood glucose levels was determined by GOD-POD Method.

Hbalc was determined by Immunoturbidometry.

Vitamin D was determined by ELISA method.

Oxidative stress (malonylaldehyde) was measured by manual TBARS(Thiobarbituricacid reactive substances method)

Cognition was assessed using Montreal cognitive assessment questionnaire.

All tests were carried out at the central lab of Sree Balaji Medical College and Hospital,Chennai.

Results were analysed using Microsoft excel.

Results: A negative correlation was seen between vitamin D and MOCA scores.

A positive correlation was seen between MDA and MOCA scores.

Conclusion: Vitamin D deficiency causes oxidative stress and affects cognition in diabetics. Vitamin D supplementation can be considered to reduce oxidative stress and thus improve cognition in diabetes mellitus patients.

Keywords: Diabetes, oxidative stress, vitamin D, Montreal cognitive assessment.

\section{Corresponding Author:}

Dr. Pushpa K.

Associate Professor in Physiology, Sridevi Institute of

Medical Sciences and Research Hospital, Tumkur 


\section{Introduction}

Diabetes is a very common disease that affects almost all body systems. Of late recent studies have determined that vitamin D deficiency can cause several diseases such as diabetes, cardiovascular complications etc. Oxidative stress is an imbalance between free radicals and antioxidants in the body. Vitamin $\mathrm{D}$ is an antioxidant and its deficiency can cause oxidative stress. ${ }^{1}$ Thus vitamin D deficiency by itself and by causing oxidative stress can increase risk of developing diabetes

Hyperglycemia can affect cognition. Oxidative stress too can affect cognition. Studies done studying the role of vitamin $\mathrm{D}$ affecting cognition especially in diabetics are very few.

Diabetes is characterized by hyperglycemia resulting from defects in insulin secretion, insulin action, or both. The chronic hyperglycemia of diabetes is associated with long-term dysfunction and failure of different organs, especially the eyes, kidneys, nerves, heart, and blood vessels. Recent studies have shown that diabetic patients are having low level of cognitive function in the form of mild cognitive impairment (MCI) which can lead to dementia and Alzheimer's disease.

\section{MCI is diagnosed when there is:}

(1) Evidence of memory impairment.

(2) Preservation of general cognitive and functional abilities.

(3) Absence of diagnosed dementia

\section{Study Design:}

Comparative cross sectional study

No of Patients data Collected $=100$ Nos

Diabetes Patients with no cognitive impairement data Collected $=43$ Nos

Diabetes Patients with mild cognitive impairement data Collected $=57$ Nos

\section{Materials and Method}

Ethical approval was obtained from the institution ethical committee.

$5 \mathrm{ml}$ of blood was taken in fasting state from the diabetes patients and subjected to following tests.

\section{Plasma Glucose Level Estimation:}

GOD - POD Method:

Principle: Glucose is oxidized by glucose oxidase (GOD) to produce gluconate and hydrogen peroxide. The hydrogen peroxide is coupled oxidatevely with 4 amino- antipyrene (4-AAP) and phenol in the presence of peroxidase (POD) to yield a red quinoeimine dye that is measured at $505 \mathrm{~nm}$. The concentration of glucose is proportional to the absorbance at $505 \mathrm{~nm}$.

$$
\begin{array}{lr}
\text { Glucose }+2 \mathrm{H}_{2} \mathrm{O}+\mathrm{O}_{2} & \text { Gluconate }+\mathrm{H}_{2} \mathrm{O}_{2} \\
2 \mathrm{H}_{2} \mathrm{O}_{2}+4 \text {-AAP }+ \text { Phenol } & \text { Quinoeimine Dye }
\end{array}
$$

Absorbance of the colored solution at $505 \mathrm{~nm}$ is directly proportional to the glucose concentration.

(Reference Range: 70 - 110 mg/d)

s Vitamin D Level Estimation: Serum vitamin D levels estimated by immunoenzymetric assay (ELISA).

\section{Principle:}

1. First 2 hours incubation step -vit.D present in calibrators,controls,samples is dissociated from binding serum proteins to fix on binding sites of a specific monoclonal antibody.

2. After 1 washing step a fixed amount of $25 \mathrm{Oh}$ vitamin $D$ labelled with biotin in presence of horsedish peroxidase (HRP),compete with unlabelled $25 \mathrm{OH}$ vitamin $\mathrm{d} 2$ and $25 \mathrm{OH}$ vitamin $\mathrm{D} 3$ present on binding sites of the specific monoclonal antibody.

3. After $30 \mathrm{~min}$ incubation at room temperature the microtiter plate is washed to stop the competition reaction.

The DIA source $25 \mathrm{OH}$ vitamin D total ELISA is a solid phase enzyme linked immunosorbent assay performed on microtiter plates.

4. The chromogenic solution is added and incubated for 15 mins.

The reaction is then stopped with the addition of stop solution and the microtiter plate is then read at the appropriate wavelength.

5. The amount of substrate turnover is determined colourimetricaly by measuring the absorbance, which is inversely proportionaly to the total $25 \mathrm{OH}$ vitamin D(D2 and D3) concentration.

(Reference range: 30 - $150 \mathrm{ng} / \mathrm{ml}$ ) 


\section{HbA1c Level Estimation}

HbA1c Level estimated by Immunoturbidimetry

Principle - Competitive binding

Reference Values:

Good control (5.6\% - 7.0\%)

Fair control $(7.01 \%-8.0 \%)$

Unsatisfactory control (8.01\% - 10.0\%)

Poor control $(>10.01 \%)$

\section{Estimation of Malonylaldehyde}

$2 \mathrm{~mL}$ of blood was collected .Itwas centrifuged at $3000 \mathrm{rpm}$ for $5 \mathrm{~min}$. Then the serum MDA was measured using the method of Buege (1978).

The samples are kept in boiling water bath for 15 $\min$.

To the diluted sample $1 \mathrm{~mL}$ of Trichloroacetic acid TCA-2-thiobarbituric acid (TBA)- $\mathrm{HCl}$ reagent is added. The reaction mixture is cooled and centrifuged. The supernatant is taken and the optical density of the pink colour formed is read at $535 \mathrm{~nm}$.
The concentration of MDA in the sample is got by plotting the obtained absorbance against the standard graph. (normal range- 2.02-4.65 $\mu \mathrm{M} / \mathrm{L}$.)

\section{Assessment of Cognition}

Overview of the MoCA

Takes approximately 15 minutes to administer

Requires informed consent

It is a screening tool and not diagnostic

MoCA Scoring

- The total points was added.

- One point was added if the patient had less than 12 years of formal education. •

- Normal score is equal to or greater than $26 / 30$.

\section{Results}

All data was analysed using microsoft excel.

Unpaired student $\mathrm{t}$ test was performed. $\mathrm{P}$ value $<0.05$ was considered significant.

Table-1: Demographic and Clinical Charecteristics of Total Type 2 Diabetic Patients with and Without MCI

\begin{tabular}{|l|c|c|c|}
\hline Number of Patients Male/Female & NO MCI N=43(43\%) (28/15) & MCI N=57(57\%) (19/38) & P Value \\
\hline Age (Years) & $48.55 \pm 7.651$ & $57.80 \pm 5.664$ & $<0.05$ \\
\hline Duration of Diabetes (Months) & $40.11 \pm 36.198$ & $156.42 \pm 8.950$ & $<0.05$ \\
\hline RBS (mg\%) & $164.34 \pm 38.999$ & $210.12 \pm 49.079$ & $<0.05$ \\
\hline HbA1c (\%) & $7.93 \pm 0.462$ & $9.18 \pm 1.137$ & $<0.05$ \\
\hline Serum vitamin D level (ng/ml) & $41.17 \pm 11.544$ & $21.97 \pm 7.063$ & $<0.05$ \\
\hline MOCA Score & $28.16 \pm 1.252$ & $20.70 \pm 2.456$ & $<0.05$ \\
\hline MDA Levels & $0.93 \pm 0.39$ & $2.65 \pm 1.33$ & $<0.05$ \\
\hline
\end{tabular}

Table 2: Correlations of MOCA Score with Other Parameters in Total Patients with MCI (n=57)

\begin{tabular}{|l|c|c|}
\hline & \multicolumn{2}{|c|}{ MOCA Score } \\
\hline & R & P \\
\hline Log10[25(OH)d] & +0.512 & 0.001 \\
\hline Duration of DM in months & -0.103 & 0.444 \\
\hline RBS (mg\%) & -0.062 & 0.647 \\
\hline HbA1c $(\%)$ & +0.003 & 0.984 \\
\hline MDA & +0.40 & 0.001 \\
\hline
\end{tabular}


Table 3: Comparision of Male and Female Patients with MCI

\begin{tabular}{|l|c|c|c|}
\hline Number of Patients & Male $(\mathbf{n}=\mathbf{1 9})$ & Female (n=38) & P Value \\
\hline Age (Years) & $58.68 \pm 4.308$ & $57.36 \pm 6.240$ & 0.412 \\
\hline Duration of DM (months) & $167.36 \pm 50.354$ & $150.94 \pm 62.719$ & 0.326 \\
\hline RBS (mg\%) & $214.73 \pm 49.252$ & $207.81 \pm 49.489$ & 0.620 \\
\hline HbA1C (\%) & $9.24 \pm 1.039$ & $9.15 \pm 1.195$ & 0.781 \\
\hline Serum Vitamin D Level (NG/ML) & $26.57 \pm 5.695$ & $19.68 \pm 6.592$ & 0.0001 \\
\hline MOCA Score & $21.73 \pm 2.921$ & $20.18 \pm 2.038$ & 0.023 \\
\hline MDA Level & $2.67 \pm 0.45$ & $2.98 \pm 0.53$ & 0.014 \\
\hline
\end{tabular}

Table 4: Average MOCA Score in All the Patients According to Different Serum Vitamin D Levels

\begin{tabular}{|c|c|c|c|}
\hline Range of serum vitamin D (ng/ml) & Average serum vitamin D level (ng/ml) & Average MOCA score & P value \\
\hline$<20(\mathrm{n}=28)$ & $15.9 \pm 2.916$ & $19.71 \pm 4.243$ & 0.0001 \\
\hline $20-30(\mathrm{n}=28)$ & $25.79 \pm 5.187$ & $22.64 \pm 3.234$ & 0.009 \\
\hline$>30(\mathrm{n}=44)$ & $42.18 \pm 10.09$ & $27.382 \pm 2.470$ & 0.0001 \\
\hline
\end{tabular}

Table 5: Association of Serum Vitamin D Level \& Moca Score in Patients with MCI in Two Age Groups

\begin{tabular}{|c|c|c|c|}
\hline Age Group & Serum vit.D $(\mathbf{n g} / \mathbf{m l})$ & MOCA score & P Value \\
\hline$<50 \mathrm{yrs}$ & $23.51 \pm 13.454$ & $21.28 \pm 3.400$ & 0.43 \\
\hline$>50 \mathrm{yrs}$ & $21.76 \pm 11.766$ & $20.62 \pm 3.866$ & 0.04 \\
\hline
\end{tabular}

\section{Discussion}

In our study, $\mathrm{HbA} 1$ cpositively correlated with MOCA scores. In a study by Roy et al., cognitive impairment was observed in $11.6 \%$ of the patients who had optimal glycemic control (HbAlc under 7\%) and $30.2 \%$ with $\mathrm{HbA} 1 \mathrm{c} 7 \%$ or above. ${ }^{5}$ Khullar et al. showed that subjects having glucose levels $>125 \mathrm{mg} / \mathrm{dl}$ had 1.73 times higher risk of developing neurocognitive impairment. ${ }^{[6,7]}$ ACCORD-MIND trial done on 2977 type 2 diabetes subjects found a statistically significant age-adjusted association between $\mathrm{HbA} 1 \mathrm{c}$ level and score on four cognitive tests. ${ }^{10}$ Both clock in a box and clockdrawing test have been shown to inversely correlate with HbA1c. ${ }^{11}$ Hence, our results are consistent with existing literature that poor glycemic control in type 2 diabetes is associated with cognitive decline.

The diabetes control and complications trial in type 1 diabetes demonstrated that improved $\mathrm{HbA} 1 \mathrm{c}$ was related to improved cognition in nonamnestic domains. ${ }^{12}$ Luchsinger et al. showed that improving $\mathrm{HbA1c}$ levels in an elderly population over a period of 5 years was associated with slowing down of global cognitive decline. ${ }^{13}$ Being a woman and longer duration of diabetes have been shown to be independent risk factors in previous studies. ${ }^{6}$ Our study did not find any difference between sex or any relation to duration of diabetes perhaps because of inadequate sample size. The MoCA is now accepted as an excellent tool for brief cognitive screening measure and is freely available with multiple editions in various languages. The original MoCA reported a sensitivity of $100 \%$ and specificity of $87 \%$ in detecting mild $\mathrm{AD}$ using a cutoff score of $26 .{ }^{9}$ Amnestic MCI (a MCI) is said to have a high likelihood of progressing to $\mathrm{AD} .{ }^{10}$ Hence, the differences noted in the MOCA scoring in our study could be suggestive of risk for development of $\mathrm{AD}$ in the future. ${ }^{15} \mathrm{~A}$ study on the effectiveness of cognitive training program in people with MCI underlines the importance of early detection of MCI. ${ }^{16}$. In summary, our study shows a high prevalence of undetected MCI in type 2 diabetes mellitus patients attending an outpatient clinic setting. A strong negative correlation was noticed between all parameters of glycemic control and MOCA scores representative of cognitive function. These observations make a strong case for routine screening of type 2 
diabetes mellitus patients to detect MCI with a sensitive tool such as MoCA. Studies on the benefits of improved glycemic control on cognitive function would need to be performed in the future to help us understand the significance of our finding in the long-term management of these patients.

Vitamin D and Oxidative Stress: When vitamin D status is adequate, many of the intracellular oxidative stress-related activities are downregulated. Having suboptimal concentrations of serum $25(\mathrm{OH})$ D fails to subdue oxidative stress conditions, augment intracellular oxidative damage and the rate of apoptosis. The intracellular Nrf2 level is inversely correlated with the accumulation of mitochondrial ROS ${ }^{17}$ and the consequent escalation of oxidative stress. Thus, Nrf2 plays a key role in protecting cells against oxidative stress; this is modulated by vitamin $\mathrm{D}$. In addition, vitamin $\mathrm{D}$ supports cellular oxidation and reduction (redox) control by maintaining normal mitochondrial functions ${ }^{29-31}$. Loss in the redox control of the cell cycle may lead to aberrant cell proliferation, cell death, the development of neurodegenerative diseases, and accelerated aging ${ }^{31-35}$. Peroxisome proliferator-activated receptor-coactivator $1 \alpha$ (PGC- $1 \alpha)$ is bound to mitochondrial deacetylase (SIRT3). PGC-1 $\alpha$ directly couples to the oxidative stress cycle ${ }^{31}$ and interacts with Nrf2. This complex regulates the expression of SIRT3; this process is influenced by vitamin D metabolites ${ }^{32}$. Calcitriol has overarching beneficial effects in upregulating the expression of certain antioxidants and anti-inflammatory cytokines ${ }^{33}$, thereby protecting the tissues from toxins, micronutrient deficiency-related abnormalities, and parasitic and intracellular microbe-induced harm ${ }^{34}$. It regulates ROS levels through its anti-inflammatory effects and mitochondrial-based expression of antioxidants through cell-signaling pathways ${ }^{34}$.In our study there was a positive correlation between vitamin $\mathrm{D}$ levels and MOCA scores. A positive correlation was also seen between HbA1c, MDA levels and MOCA scores.

Supplementation of vitamin D in Diabetic patients can prove to be beneficial in reducing oxidative stress, improving cognition as well as help control blood sugar levels.

\section{Interests of Conflict: None}

Funding: Self

\section{References}

1. Contreras CM, Gutiérrez-García AG. Cognitive impairment in diabetes and poor glucose utilization in the intracellular neural milieu. Med Hypotheses 2017; 104:160-5

2. Whitlow CT, Sink KM, Divers J, Smith SC, Xu J, Palmer ND, et al. Effects of type 2 diabetes on brain structure and cognitive function: African Americandiabetes heart study MIND. Am J Neuroradiol 2015; 36:1648-53.

3. Zilliox LA, Chadrasekaran K, Kwan JY, Russell JW. Diabetes and cognitive impairment. Curr Diab Rep 2016; 16:87.

4. Velayudhan L, Ryu SH, Raczek M, Philpot M, Lindesay J, Critchfield M, et al. Review of brief cognitive tests for patients with suspected dementia. Int Psychogeriatr 2014; 26:1247-62.

5. Roy S, Kim N, Desai A, Komaragiri M, Baxi N, Jassil $\mathrm{N}$, et al. Cognitive function and control of type 2 diabetes mellitus in young adults. N Am J Med Sci 2015; 7:220-6.

6. Khullar S, Kaur G, Dhillon H, Sharma R, Mehta K, Singh M, et al. The prevalence and predictors of cognitive impairment in type 2 diabetic population of Punjab, India. J Soc Health Diabetes 2017; 5:4753.

7. Solanki, R K., Dubey V, Munshi, D. Neurocognitive impairment and comorbid depression in patients of diabetes mellitus. Int J Diabetes Dev Ctries 2009; 29:133-8.

8. Chen RH, Jiang XZ, Zhao XH, Qin YL, Gu Z, Gu PL, et al. Risk factors of mild cognitive impairment in middle aged patients with type 2 diabetes: A cross-section study. Ann Endocrinol (Paris) 2012; 73:208-12.

9. Nasreddine ZS, Phillips NA, Bédirian V, Charbonneau S, Whitehead V, Collin I, et al. The Montreal Cognitive Assessment, MoCA: A brief screening tool for mild cognitive impairment. J Am Geriatr Soc 2005; 53:695-9.

10. Cukierman-Yaffe T, Gerstein HC, Williamson JD, Lazar RM, Lovato L, Miller ME, et al. Relationship between baseline glycemic control and cognitive function in individuals with type 2 diabetes and other cardiovascular risk factors: The action to control cardiovascular risk in diabetes-memory in diabetes (ACCORD-MIND) trial. Diabetes Care 2009; 32:221-6. 
11. Sukontapol C, Kemsen S, Chansirikarn S, Nakawiro D, Kuha O, Taemeeyapradit U. The effectiveness of a cognitive training program in people with mild cognitive impairment: A study in urban community. Asian J Psychiatr 2018; 35:61-6.

12. Holmes, S.; Abbassi, B.; Su, C.; Singh, M.; Cunningham, R.L. Oxidative stress defines the neuroprotective or neurotoxic properties of androgens in immortalized female rat dopaminergic neuronal cells. Endocrinology 2013; 154: 4281-92.

13. Petersen, K.S.; Smith, C. Ageing-Associated Oxidative Stress and Inflammation Are Alleviated by Products from Grapes. Oxid. Med. Cell. Longev. 2016; 2016: 6236309.

14. Nakai, K.; Fujii, H.; Kono, K.; Goto, S.; Kitazawa, R.; Kitazawa, S.; Hirata, M.; Shinohara, M.; Fukagawa, M.; Nishi, S. Vitamin D activates the Nrf2-Keap1 antioxidant pathway and ameliorates nephropathy in diabetic rats. Am. J. Hypertens. 2014; 27:586-95.

15. Lewis, K.N.; Mele, J.; Hayes, J.D.; Buffenstein, R. Nrf2, a guardian of healthspan and gatekeeper of species longevity. Integr. Comp. Biol. 2010; 50: 829-43.

16. Tullet, J.M.A.; Green, J.W.; Au, C.; Benedetto, A.; Thompson, M.A.; Clark, E.; Gilliat, A.F.; Young, A.; Schmeisser, K.; Gems, D.TheSKN-1/Nrf2 transcription factor can protect against oxidative stress and increase lifespan in C. elegans by distinct mechanisms. Aging Cell 2017; 16:1191-94.

17. Forster, R.E.; Jurutka, P.W.; Hsieh, J.C.; Haussler, C.A.; Lowmiller, C.L.; Kaneko, I.; Haussler, M.R.; Kerr Whitfield, G. Vitamin D receptor controls expression of the anti-aging klotho gene in mouse and human renal cells. Biochem. Biophys. Res. Commun. 2011; 414:557-62.

18. Berridge, M.J. Vitamin D: A custodian of cell signalling stability in health and disease. Biochem. Soc. Trans. 2015; 43:349-358.

19. Razzaque, M.S. FGF23, klotho and vitamin D interactions: What have we learned from in vivo mouse genetics studies? Adv. Exp. Med. Biol. 2012; 728: 84-91.

20. Kuro-o, M. Klotho and aging. Biochim. Biophys. Acta 2009; 1790:1049-58.

21. Tseng, A.H.; Shieh, S.S.; Wang, D.L. SIRT3 deacetylates $\mathrm{FOXO} 3$ to protect mitochondria against oxidative damage. Free Radic. Biol. Med.
2013; 63:222-34.

22. Wang, L.; Lewis, T.; Zhang, Y.L.; Khodier, C.; Magesh, S.; Chen, L.; Inoyama, D.; Chen, Y.; Zhen, J.; Hu, L.; etal. The identification and characterization of non-reactive inhibitor of Keap1Nrf2 interaction through HTS using a fluorescence polarization assay. In Probe Reports from the NIH Molecular Libraries Program; National Center for Biotechnology Information (US): Bethesda, MD, USA, 2010.

23. Berridge, M.J. Vitamin D deficiency: Infertility and neurodevelopmental diseases (attention deficit hyperactivity disorder, autism, and schizophrenia). Am. J. Physiol. Cell Physiol. 2018; 314:C135-151.

24. Ryan, Z.C.; Craig, T.A.; Folmes, C.D.; Wang, X.; Lanza, I.R.; Schaible, N.S.; Salisbury, J.L.; Nair, K.S.; Terzic, A.; Sieck, G.C.; et al. 1alpha, 25-Dihydroxyvitamin D3 Regulates Mitochondrial Oxygen Consumption and Dynamics in Human Skeletal Muscle Cells. J. Biol. Chem. 2016; 291:1514-28.

25. Bouillon, R.; Verstuyf, A. Vitamin D, mitochondria, and muscle. J.Clin. Endocrinol. Metab. 2013; 98:961-63.

26. Sarsour, E.H.; Kumar, M.G.; Chaudhuri, L.; Kalen, A.L.; Goswami, P.C. Redox control of the cell cycle in health and disease. Antioxid. Redox Signal. 2009; 11:2985-11.

27. Lin, M.T.; Beal, M.F. Mitochondrial dysfunction and oxidative stress in neurodegenerative diseases. Nature 2006; 443:787-95.

28. Berridge, M.J. Vitamin D cell signalling in health and disease. Biochem. Biophys. Res. Commun. 2015; 460:53-71.

29. Ureshino, R.P.; Rocha, K.K.; Lopes, G.S.; Bincoletto, C.; Smaili, S.S. Calcium signaling alterations, oxidative stress, and autophagy in aging. Antioxid. Redox Signal. 2014; 21:123-37.

30. Chen, Y.; Zhang, J.; Lin, Y.; Lei, Q.; Guan, K.L.; Zhao, S.; Xiong, Y.Tumoursuppressor SIRT3deacetylates and activates manganese superoxide dismutase to scavenge ROS. EMBO Rep. 2011; 12:534-41.

31. Song, C.; Fu, B.; Zhang, J.; Zhao, J.; Yuan, M.; Peng, W.; Zhang, Y.; Wu, H. Sodium fluoride induces nephrotoxicity via oxidative stressregulated mitochondrial SIRT3 signaling pathway. Sci. Rep. 2017; 7:72. 
32. Wei, R.; Christakos, S. Mechanisms Underlyingthe Regulation of Innateand Adaptive Immunity by Vitamin D. Nutrients 2015; 7:8251-60.

33. George, N.; Kumar, T.P.; Antony, S.; Jayanarayanan, S.; Paulose, C.S. Effect of vitamin D3 in reducing metabolic and oxidative stress in the liver of streptozotocin-induced diabetic rats. Br. J. Nutr. 2012; 108:1410-18.
34. Shelton, R.C.; Claiborne, J.; SidorykWegrzynowicz, M.; Reddy, R.; Aschner, M.; Lewis, D.A.; Mirnics, K. Altered expression of genes involved in inflammation and apoptosis in frontal cortex in major depression. Mol. Psychiatry 2011; 16:751-62. 